Quality filter:

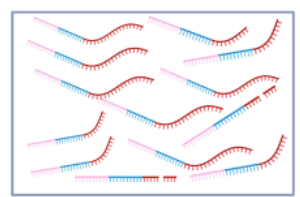

Adapter trimming Primer trimming

Length filter

Phred score filter

Alignment to the reference

Mapping reads only retained
Mapping:

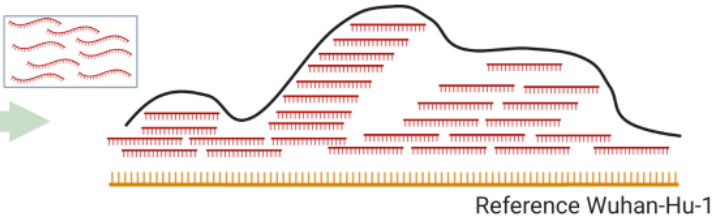

Coverage filter :

only positions covered by more than 50 reads are considered highly covered

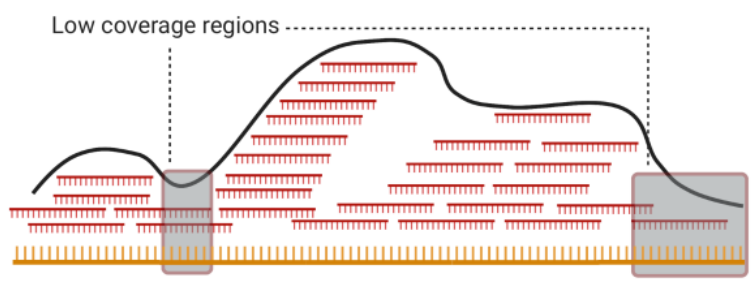

Consensus sequence generation:

Called mutation

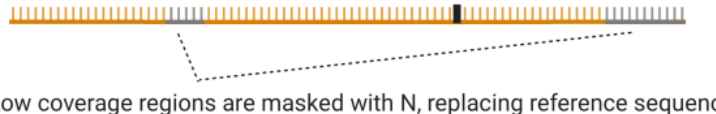

Calling only highly read-supported variants only mutations supported by $70 \%$ of available reads and not in low covered regions are retained

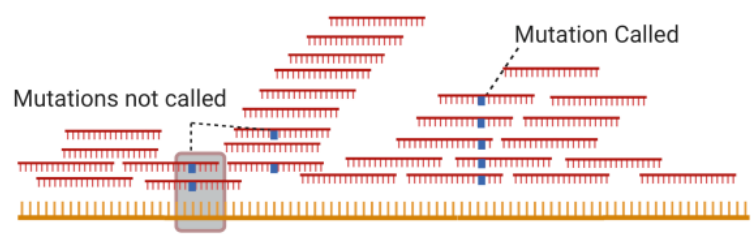

\section{QC checking:}

sequences with $>10 \%$ Ns are discarded

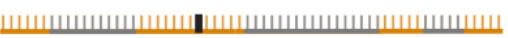

QC checking:

sequences with $<10 \%$ Ns are retained

Figure S4. The COVGAP pipeline. The steps shown ensure the calculation of high quality consensus sequences. Particularly, information on read coverage is retained and used both in the variant calling procedure and in the draft of the consensus independently from the called variants. Finally, the quality of the genome from each sample is scored by \%Ns, which determines whether the produced sequence is retained or discarded. Support for the creation of this schematic figure was provided by BioRender.com. 